Received: 25.06 .2020

Revised: 22.07 .2020

Accepted: 21.08 .2020

DOI: $10.17804 / 2410-9908.2020 .4 .062-078$

\title{
STUDYING THE STRATIFICATION OF HYDRODYNAMIC FIELDS FOR LAMINAR FLOWS OF VERTICALLY SWIRLING FLUID
}

\author{
N. V. Burmasheva ${ }^{a)^{*}}$ and E. Yu. Prosviryakov ${ }^{\text {b) }}$ \\ Institute of Engineering Science, Ural Branch of the Russian Academy of Sciences, \\ 34, Komsomolskaya St., Ekaterinburg, 620049, Russian Federation \\ a) (iD https://orcid.org/0000-0003-4711-1894 $\otimes$ nat_burm@mail.ru; \\ b) (iD https://orcid.org/0000-0002-2349-7801 《 evgen_pros@mail.ru \\ *Corresponding author. E-mail: nat_burm@mail.ru \\ Address for correspondence: 34 Komsomolskaya St., Ekaterinburg, 620049, Russian Federation \\ Tel.:+7 (343) 37535 76; fax: 3745330
}

The article proposes an approach to estimating the number of stratification points in hydrodynamic fields. The article provides a method allowing one to estimate from above the number of zero points of hydrodynamic fields (points and stratification zones). The application of the proposed methodology is illustrated by several examples of the analysis of the exact solution to the problem of describing steady laminar flows of a viscous incompressible fluid in an infinite horizontal layer. In example 1, convection is induced by setting the shear stress field at one of the layer boundaries. The features of the background temperature profile, which is a seventh-degree polynomial, are discussed. It is shown that this component of the temperature field is a nonmonotonic function and that the obtained exact solution for the temperature field can describe the stratification of the considered fluid layer into one, two or three zones relative to the reference value. Example 2 illustrates evaluating the number of the zero points of the velocity field components in a vertically swirling fluid, in which convective flows are initiated by thermocapillary forces at the upper boundary of the layer. The exact solution studied in this example is a sixth-degree polynomial, which can have at most two zeros inside the region under consideration. This means that this exact solution is able to describe the stratification of the fluid layer into three zones, in each of which the test speed takes values of the same sign.

Keywords: laminar flow, vertically swirling fluid, exact solution, thermocapillary convection, tangential stresses, zero field points, field stratification.

\section{References}

1. Nalivkin D.V. Uragani, buri i smerchi [Hurricanes, Storms and Tornadoes]. Leningrad, Nauka, 1969, 487 p. (In Russian).

2. Monin A.S., Kamenkovich V.M. Okeanologiya. Fizika okeana. T. I. Gidrofizika okeana [Oceanology. Physics of the ocean. Vol. I: Ocean Hydrophysics]. Moscow, Nauka, 1987, 466 p. (In Russian).

3. Alekseenko S.V., Kuybin P.A., Okulov V.L. Vvedenie v teoriyu koncentrirovannikh vikhrey [Introduction to Concentrated Vortex Theory]. Novosibirsk, Institut teplofiziki im. S.S. Kutateladze Publ., 2003, 504 p. (In Russian).

4. Lavrent'ev M.A., Shabat B.V. Problemi gidrodinamiki i ikh matematicheskie modeli [Problems of Hydrodynamics and their Mathematical Models, 2-nd education]. Moscow, Nauka, 1973, 407 p. (In Russian). 
5. Burmasheva N.V., Prosviryakov E.Yu. A large-scale layered stationary convection of an incompressible viscous fluid under the action of shear stresses at the upper boundary. Velocity field investigation. Vestn. Sam. gos. tekh. univer. Ser. Fiz.-mat. nauki, 2017, vol. 21, no. 1, pp. 180-196. DOI: 10.14498/vsgtu1527 (In Russian).

6. Shvarts K.G. Plane-parallel advective flow in a horizontal layer of an incompressible fluid with an internal linear heat source. Fluid Dynamics, 2018, vol. 53, no. 1, pp. S24-S28. DOI: $10.1134 / \mathrm{S} 0015462818040237$.

7. Burmasheva N.B., Prosviryakov E.Yu. Exact solution for the layered convection of a viscous incompressible fluid at specified temperature gradients and tangential forces on the free boundary. AIP Conference Proceedings, 2017, vol. 1915, iss. 1. DOI: 10.1063/1.5017353.

8. Burmasheva N.V., Prosviryakov E.Yu. Convective layered flows of a vertically whirling viscous incompressible fluid. Velocity field investigation. Vestn. Sam. gos. tekh. univer. Ser. Fiz.mat. nauki, 2019, vol. 23, no. 2, pp. 341-360. DOI: 10.14498/vsgtu1670.

9. Burmasheva N.B., Prosviryakov E.Yu. Investigation of a velocity field for the Marangoni shear convection of a vertically swirling viscous incompressible fluid. AIP Conference Proceedings, 2018, vol. 2053, iss. 1. DOI: 10.1063/1.5084449.

10. Bekezhanova V.B., Rodionova A.V. Longwave stability of two-layer fluid flow in the inclined plane. Fluid Dynamics, 2015, vol. 50, no. 6, pp. 723-736. DOI: 10.1134/S0015462815060010.

11. Andreyev V.K., Bekezhanova V.B. The free-parameter solution of the convection equations in a vertical cylinder with a volume heat source. Journal of Applied Mathematics and Mechanics, 2013, vol. 77, no. 6, pp. 595-602. DOI: 10.1016/j.jappmathmech.2014.03.004.

12. Burmasheva N.B., Larina E.A., Prosviryakov E.Yu. Unidirectional convective flows of a viscous incompressible fluid with slippage in a closed layer. AIP Conference Proceedings, 2019, vol. 2176, iss. 1. DOI: 10.1063/1. 5135147.

13. Burmasheva N.B., Prosviryakov E.Yu. Temperature field investigation in layered flows of a vertically swirling viscous incompressible fluid under two thermocapillar forces at a free boundary. DReaM, 2019, iss. 1, pp. 6-42. DOI: 10.17804/2410-9908.2019.1.006-042.

14. Burmasheva N.V., Prosviryakov E. Yu. Thermocapillary convection of a vertical swirling liquid. Theor. Found. Chem. Eng., 2020, vol. 54, no. 1, pp. 230-239. DOI: 10.1134/S0040579519060034.

15. Bekezhanova V.B. Convective instability of Marangoni-Poiseuille flow under a longitudinal temperature gradient. Journal of Applied Mechanics and Technical Physics, 2011, vol. 52, no. 1, pp. 74-81. DOI: 10.1134/S0021894411010111.

16. Burmasheva N.V., Prosviryakov E.Yu. Exact solution of Navier-Stokes equations describing spatially inhomogeneous flows of a rotating fluid. Trudy Instituta Matematiki $i$ Mekhaniki UrO RAN, 2020, vol. 26, no. 2. pp. 79-87. (In Russian).

17. Birikh R.V., Pukhnachev V.V., Frolovskaya O.A. Convective flow in a horizontal channel with non-newtonian surface rheology under time-dependent longitudinal temperature gradient. Fluid Dynamics, 2015, vol. 50, no. 1, pp. 173-179. DOI: 10.1134/S0015462815010172.

18. Burmasheva N.V., Prosviryakov E.Yu. A class of exact solutions for two-dimensional equations of geophysical hydrodynamics with two Coriolis parameters. The Bulletin of Irkutsk State University. Series Mathematics, 2020, vol. 32, pp. 33-48. DOI: 10.26516/1997-7670.2020.32.33. (In Russian).

19. Goncharova O.N. Two-layer fluid flows with evaporation at an interface in the presence of an anomalous thermocapillary effect. Izvestiya of Altai State University, 2015, no. 1-2 (85), pp. 101-105. DOI: 10.14258/izvasu(2015)1.2-18. (In Russian). 
20. Aleksandrov A.D., Kolmogorov A.N., Lavrent'ev M.A. Matematika, eyo soderganie, metody $i$ znachenie. T. 1 [Mathematics, its contents, methods and meaning. Vol. 1]. Moscow, Izdatelstvo Akademii nauk SSSR Publ., 1956, 536 p. (In Russian).

21. Shafarevich I.R. O reshenii uravneniy vishikh poryadkov (metod Shturma) [On solving equations of higher degrees (Sturm method)]. Moscow, Gostekhizdat Publ., 1954, 24 p. (In Russian).

22. Bashmakova I.G. On the proof of fundamental theorem of algebra. In: Istorikomatematicheskie issledovaniya [Historical and Mathematical Research. Issue 10]. Moscow, GITL Publ., 1957, pp. 257-305. (In Russian).

23. Demidovich B.P. Lekcii po matematicheskoy teorii ustoychivosti: uchebnoe posobie [Lectures on Mathematical Theory of Stability: Educational book]. Sankt-Peterburg, Lan', 2008, 480 p. (In Russian).

24. Petrov A.G. Exact solution of the Navier-Stokes equations in a fluid layer between the moving parallel plates. Journal of Applied Mechanics and Technical Physics, 2012, vol. 53, no. 5, pp. 642-646. DOI: 10.1134/S0021894412050021.

25. Shmiglevskiy Yu.D. Analiticheskie issledovaniya dinamiki zhidkosti i gaza [Analytical Studies of Fluid and Gas Dynamics]. Moscow, Editorial URSS, 1999, 232 p. (In Russian).

26. Kochin N.E., Kibel' I.A., Roze N.B. Teoreticheskaya gidrodinamika. Chast' 2. [Theoretical Hydrodynamics. Part 2]. Moscow: FIZMATLIT Publ., 1963, 728 p. (In Russian).

27. Loytsyanskiy L.G. Mekhanika zhidkosti $i$ gaza [Fluid and Gas Mechanics]. M. : DROFA Publ., 2003, 840 p. (In Russian).

28. Shvarts K.G. Advective Flow of a rotating fluid layer in a vibrational field. Russian Journal of Nonlinear Dynamics, 2019, vol. 15, no. 3, pp. 261-270. DOI: 10.20537/nd190305.

29. Aristov S.N., Shvarts K.G. Advective flow in a rotating liquid film. Journal of Applied Mechanics and Technical Physics, 2016, vol. 57, no. 1, pp. 188-194. DOI: 10.1134/S0021894416010211.

30. Gershuni G.Z., Zhukhovitskii E.M. Convective Stability of Incompressible Fluids [Original Russian text published in Gershuni G.Z., Zhukhovitskii E.M. Konvektivnaya ustoichivost' neszhimaemoy zhidkosti. Moscow, Nauka, 1972, 392 p.], Israel Program for Scientific Translations, Jerusalem, Keter Publishing House, 1976, 330 p.

31. Landau L.D., Lifshitz E.M. Fluid Mechanics (Volume 6 of A Course of Theoretical Physics) [Original Russian text published in Landau L.D., Lifshitz E.M. Teoreticheskaya fizika. Tom 6. Gidrodinamika. Moscow, FIZMATLIT, 1986, 736 p.], Oxford, Pergamon Press, 1987, 536 p.

32. Boussinesq J. Theorie analitique de la chaleur. Vol. 2, Paris, GauthierVillars, 1903, 625 p.

33. Oberbeck A. Uber die warmeleitung der flussigkeiten bei der berucksichtigung der stromungen infolge von temperaturdifferenzen. Annal. Phys. Chem., 1879, bd. 7, № 6, s. 271-292.

34. Aristov S.N., Prosviryakov E.Yu. Nonuniform convective Couette flow. Fluid Dynamics, 2016, vol. 51, no. 5, p. 581-587. DOI: 10.1134/S001546281605001X.

35. Aristov S.N., Prosviryakov E.Y. On laminar flows of planar free convection. Rus. J. Nonlin. Dyn., 2013, vol. 9, no. 4, pp. 651-657. DOI: 10.20537/nd1304004. (In Russian).

36. Zaytsev M.L., Akkerman V.B. Hypothesis on reduction of overdetermined systems of differential equations and its application to equations of hydrodynamics. Vestn. Voronezh. gos. univer. Ser.: Fizika. Matematika, 2015, no. 2, pp. 5-27. (In Russian).

37. Tikhonov A.N., Samarskiy A.A. Uravneniya matematicheskoy fiziki [Equations of Mathematical Physics]. Moscow, Nauka, 1966, 742 p. (In Russian).

38. Pirov R. On compatibility conditions and manilolds of solutions to one class of overdetermined systems of second order partial differential equations. Ufa Math. Journal, 2016, vol. 8, no. 2, pp. 58-64. DOI: 10.13108/2015-0-2-58. 
39. Targ S.M. Coriolis force. In: Fizicheskaya entsiklopediya [Physical Encyclopedia. Vol. 2]. Moscow, Sovetskaya entsiklopediya Publ., 1990, 704 p. (In Russian).

40. Coriolis G. Sur les equations du mouvement relative des systemes de corps. Journ. Ecole polytechn., 1835, vol. 15, no. 24, pp. 142-154.

41. Lopez-Mariscal M. Further Coriolis correlation considerations. Physics Today, 2012, vol. 65, iss. 11, pp. 8-9. DOI: 10.1063/PT.3.1764.

42. Graney Ch.M. Coriolis effect, two centuries before Coriolis. Physics Today, 2011, vol. 64, iss. 8, pp. 8-9. DOI: 10.1063/PT.3.1195.

43. Tartishnikov E.E. Matrichniy analiz i lineynaya algebra [Matrix Analysis and Linear Algebra]. Moscow, MGU Publ., 2005, 372 p. (In Russian). 
Подана в журнал: 25.06 .2020

УДК 532.51:517.958

DOI: $10.17804 / 2410-9908.2020 .4 .062-078$

\title{
ИССЛЕДОВАНИЕ СТРАТИФИКАЦИИ ГИДРОДИНАМИЧЕСКИХ ПОЛЕЙ ДЛЯ СЛОИСТЫХ ТЕЧЕНИЙ ВЕРТИКАЛЬНО ЗАВИХРЕННОЙ ЖИДКОСТИ
}

\author{
Н. В. Бурмашева ${ }^{\text {a)* }}$ Е. Ю. Просвиряков ${ }^{\text {) }}$ \\ Институт машиноведения УрО РАН, ул. Комсомольская, \\ 34, г. Екатеринбург, Российская Федерация \\ a) iD https://orcid.org/0000-0003-4711-1894 nat_burm@ @ail.ru; \\ б) (iD https://orcid.org/0000-0002-2349-7801 《 evgen_pros@mail.ru \\ *Ответственный автор. Электронная почта: nat_burm@ @ail.ru \\ Адрес для переписки: ул. Комсомольская, 34, г. Екатеринбург, Российская Федерация \\ Тел.: +7 (343) 375-35-76; факс: 374-53-30
}

В статье предложен подход к оценке числа точек стратификации гидродинамических полей. В статье приведен метод, с помощью которого можно оценить сверху число нулевых точек гидродинамических полей (точек и зон стратификации). Применение предлагаемой методики проиллюстрировано на нескольких примерах анализа точного решения задачи описания установившихся слоистых течений вязкой несжимаемой жидкости в бесконечном горизонтальном слое. В первом из них конвекция индуцируется заданием поля касательных напряжений на одной из границ слоя. Обсуждаются особенности профиля фоновой температуры, являющейся многочленом седьмой степени. Показано, что данная компонента поля температуры является немонотонной функцией и полученное точное решение для поля температуры может описывать стратификацию рассматриваемого слоя жидкости на одну, две или три зоны относительно отсчетного значения. Второй пример посвящен оценке числа нулевых точек компонент поля скорости вертикально завихренной жидкости, конвективные течения в которой инициируются термокапиллярными силами на верхней границе слоя. Исследуемое в этом примере точное решение есть полином шестой степени, который может иметь не более двух нулей внутри рассматриваемой области. Это означает, что данное точное решение способно описывать расслоение слоя жидкости на три зоны, в каждой из которых исследуемая скорость принимает значения одного знака.

Ключевые слова: слоистое течение, вертикально завихренная жидкость, точное решение, термокапиллярная конвекция, касательные напряжения, нулевые точки поля, расслоение поля.

\section{1. Введение}

Известно [1-4], что на свойства течений жидкости значительное влияние оказывают движущиеся в них вихри, вследствие чего изучение условий генерации и структуры вихрей становится крайне важным. Однако теоретическое исследование вихрей и влияния их интенсивности на масштабы межвихревого взаимодействия далеко от завершения, потому отыскание новых точных решений, описывающих вихревые течения жидкости, является актуальным. Особый интерес среди этих решений вызывают классы точных решений, сохраняющие нелинейные свойства исходных уравнений движения жидкости.

Чаще всего точные решения, описывающие установивишиеся течения, являются полиномиальными [5-19], причем эти полиномы имеют высокую степень, что делает их анализ весьма нетривиальной задачей. Полученные точные решения являются суперпозицией нескольких монотонных полиномов, каждый из которых вносит вклад в структуру гидродинамического поля. Для оценки числа нулей многочленов с постоянными коэффициентами раз- 
работан математический аппарат линейной алгебры: теорема Декарта [20] о числе перемен знаков в ряду коэффициентов, ряд Штурма [21], основная теорема алгебры об оценке количества нулей многочлена его степенью [22], критерий Рауса-Гурвица [23] и др. Однако в случае, когда значения коэффициентов заранее неизвестны, разработанные для полиномов методы оказываются мало пригодны.

Кроме того, не всегда получаемое точное решение описывается полиномиальной зависимостью, иногда решение для исследуемого гидродинамического поля есть некоторая нелинейная функция общего вида [16, 18, 24-29]. И в таких ситуациях перечисленные общие математические методы неприменимы вовсе, при том что потребность в анализе таких решений остается.

В данной статье предложен один способ определения верхней оценки числа критических точек основных гидродинамических полей, которые могут и не описываться полиномами, а являться некоторыми нелинейными функциями. Применение предлагаемого подхода к оценке числа критических точек полей проиллюстрировано на примере точного решения системы уравнений тепловой конвекции для вертикально завихренной жидкости. Стоит отметить, что предложенный в статье подход применим и к другим задачам механики слоистых течений вязкой несжимаемой жидкости, а также к задачам из различных разделов математической физики, где возникает подобная проблема.

\section{2. Система уравнений Обербека-Буссинеска для слоистых течений}

В случае установившихся слоистых течений $\left(V_{x}(x, y, z) ; V_{y}(x, y, z) ; 0\right)$ система уравнений тепловой конвекции в приближении Буссинеска [5; 7-9; 12-14; 30-35] принимает вид:

$$
\begin{gathered}
V_{x} \frac{\partial V_{x}}{\partial x}+V_{y} \frac{\partial V_{x}}{\partial y}=-\frac{\partial P}{\partial x}+v \Delta V_{x} \\
V_{x} \frac{\partial V_{y}}{\partial x}+V_{y} \frac{\partial V_{y}}{\partial y}=-\frac{\partial P}{\partial y}+v \Delta V_{y} \\
\frac{\partial P}{\partial z}=g \beta T ; \\
V_{x} \frac{\partial T}{\partial x}+V_{y} \frac{\partial T}{\partial y}=\chi \Delta T ; \\
\frac{\partial V_{x}}{\partial x}+\frac{\partial V_{y}}{\partial y}=0 .
\end{gathered}
$$

Уравнения (1) являются проекциями векторного уравнения движения жидкости на оси декартовой системы координат $O x y z$, уравнение теплопроводности (2) описывает изменение температурного поля, а уравнение (3) есть уравнение несжимаемости. В системе (1)-(4) через $P(x, y, z)$ обозначено отклонение давления от гидростатического, деленное на постоянную среднюю плотность $\rho$ жидкости [30, 31]; $T(x, y, z)$ - отклонение от отсчетного значения температуры $[30,31] ; v, \chi, \beta, g$ - коэффициенты кинематической вязкости, температуропроводности, температурного расширения жидкости и ускорение свободного падения соответственно; $\Delta=\left(\frac{\partial^{2}}{\partial x^{2}}+\frac{\partial^{2}}{\partial y^{2}}+\frac{\partial^{2}}{\partial z^{2}}\right)-$ оператор Лапла- 
са. Отметим, что система уравнений (1)-(3) является переопределенной, так как число уравнений превосходит число неизвестных [36-38].

Точное решение системы Обербека-Буссинеска (1)-(3) будем искать в виде [8, 9, 13, $14,16,18]:$

$$
\begin{gathered}
V_{x}=U(z)+u(z) y, \quad V_{y}=V(z) ; \\
T=T_{0}+T_{1}(z) x+T_{2}(z) y, P=P_{0}(z)+P_{1}(z) x+P_{2}(z) y .
\end{gathered}
$$

Поле скоростей (4) позволяет описывать вертикально завихренную жидкость (вертикальная компонента вектора завихренности $\Omega_{z}=\frac{\partial V_{y}}{\partial x}-\frac{\partial V_{z}}{\partial y}=-u(z)$ ) вне поля силы Кориолиса [5; 7-9; 12-14; 31; 39-42]. Разрешимость системы (1)-(3) обеспечивается тем, что при подстановке класса (4) уравнение несжимаемости (3) тождественно выполняется.

Подставим выбранный класс (4) и (5) в систему уравнений тепловой конвекции (1)-(3):

$$
\begin{gathered}
(U+u y) \frac{\partial(U+u y)}{\partial x}+V \frac{\partial(U+u y)}{\partial y}= \\
=-\frac{\partial\left(P_{0}+P_{1}(z) x+P_{2}(z) y\right)}{\partial x}+v\left(\frac{\partial^{2}}{\partial x^{2}}+\frac{\partial^{2}}{\partial y^{2}}+\frac{\partial^{2}}{\partial z^{2}}\right)(U+u y) \\
(U+u y) \frac{\partial V}{\partial x}+V \frac{\partial V}{\partial y}=-\frac{\partial\left(P_{0}+P_{1} x+P_{2} y\right)}{\partial y}+v\left(\frac{\partial^{2}}{\partial x^{2}}+\frac{\partial^{2}}{\partial y^{2}}+\frac{\partial^{2}}{\partial z^{2}}\right) V ; \\
\frac{\partial\left(P_{0}+P_{1} x+P_{2} y\right)}{\partial z}=g \beta\left(T_{0}+T_{1} x+T_{2} y\right) ; \\
(U+u y) \frac{\partial\left(T_{0}+T_{1} x+T_{2} y\right)}{\partial x}+V \frac{\partial\left(T_{0}+T_{1} x+T_{2} y\right)}{\partial y}=\chi\left(\frac{\partial^{2}}{\partial x^{2}}+\frac{\partial^{2}}{\partial y^{2}}+\frac{\partial^{2}}{\partial z^{2}}\right)\left(T_{0}+T_{1} x+T_{2} y\right) \\
\frac{\partial(U+u y)}{\partial x}+\frac{\partial V}{\partial y}=0 .
\end{gathered}
$$

Откуда далее при использовании метода неопределенных коэффициентов [5; 7-9; 12-14] получаем систему обыкновенных дифференциальных уравнений для определения составляющих гидродинамических полей:

$$
\begin{gathered}
u^{\prime \prime}=0 ; T_{1}^{\prime \prime}=0 ; P_{1}^{\prime}=g \beta T_{1} ; \chi T_{2}^{\prime \prime}=u T_{1} ; P_{2}^{\prime}=g \beta T_{2} ; \\
v V^{\prime \prime}=P_{2} ; v U^{\prime \prime}=V u+P_{1} ; \chi T_{0}^{\prime \prime}=U T_{1}+V T_{2} ; P_{0}^{\prime}=g \beta T_{0} .
\end{gathered}
$$

Здесь и далее штрихом обозначена производная по вертикальной координате $z$. Уравнения системы (6) интегрируются в том порядке, в котором они выписаны.

Точное решение системы (6) является полиномиальным:

$$
T_{1}=c_{1} z+c_{2}, P_{1}=\frac{g \beta c_{1}}{2} z^{2}+g \beta c_{2} z+c_{3}, T_{2}=\frac{c_{1} c_{4}}{12 \chi} z^{4}+\frac{c_{2} c_{4}+c_{1} c_{5}}{6 \chi} z^{3}+\frac{c_{2} c_{5}}{2 \chi} z^{2}+c_{6} z+c_{7}
$$




$$
\begin{gathered}
P_{2}=\frac{g \beta c_{1} c_{4}}{60 \chi} z^{5}+\frac{g \beta\left(c_{2} c_{4}+c_{1} c_{5}\right)}{24 \chi} z^{4}+\frac{g \beta c_{2} c_{5}}{6 \chi} z^{3}+\frac{g \beta c_{6}}{2} z^{2}+g \beta c_{7} z+c_{8}, u=c_{4} z+c_{5} \\
V=\frac{g \beta c_{1} c_{4}}{2520 v \chi} z^{7}+\frac{g \beta\left(c_{2} c_{4}+c_{1} c_{5}\right)}{720 v \chi} z^{6}+\frac{g \beta c_{2} c_{5}}{120 v \chi} z^{5}+\frac{g \beta c_{6}}{24 v} z^{4}+\frac{g \beta c_{7}}{6 v} z^{3}+\frac{c_{8}}{2 v} z^{2}+c_{9} z+c_{10} \\
U=\frac{g \beta c_{1} c_{4}^{2}}{226800 v^{2} \chi} z^{10}+\frac{g \beta c_{4}\left(7 c_{2} c_{4}+9 c_{1} c_{5}\right)}{362880 v^{2} \chi} z^{9}+\frac{g \beta c_{5}\left(7 c_{2} c_{4}+c_{1} c_{5}\right)}{40320 v^{2} \chi} z^{8}+ \\
+\frac{g \beta\left(c_{2} c_{5}^{2}+5 \chi c_{4} c_{6}\right)}{5040 v^{2} \chi} z^{7}+\frac{g \beta\left(c_{5} c_{6}+4 c_{4} c_{7}\right)}{720 v^{2}} z^{6}+\frac{g \beta c_{5} c_{7}+3 c_{4} c_{8}}{120 v^{2}} z^{5}+ \\
+\frac{g \beta v c_{1}+c_{5} c_{8}+2 v c_{4} c_{9}}{24 v^{2}} z^{4}+\frac{g \beta c_{2}+c_{5} c_{9}+c_{4} c_{10}}{6 v} z^{3}+\frac{c_{3}+c_{5} c_{10}}{2 v} z^{2}+c_{11} z+c_{12} .
\end{gathered}
$$

Выражения для фоновой температуры $T_{0}$ и фонового давления $P_{0}$ здесь не приводятся ввиду их громоздкости и простоты дальнейшего интегрирования системы (6).

Для того чтобы полученное общее решение (7) описывало физические процессы, происходящие в жидкости, необходимо определить из краевых условий все возникшие в ходе интегрирования системы (6) константы. Поскольку система (6) является системой пятнадцатого порядка, требуется сформулировать пятнадцать условий, описывающих изменение основных гидродинамических характеристик на границах рассматриваемой области. Отметим, что выбор краевых условий не влияет на развиваемый в рамках статьи подход к оценке числа критических точек, а служит лишь для удобства интерпретации получаемых оценок.

\section{3. Описание методики построения оценки числа точек стратификации}

Пусть для некоторого однородного гидродинамического поля $F(z)$, изменяющегося при течении жидкости в слое $a \leq z \leq b$, получено точное решение в виде:

$$
F(z)=k_{0}+\sum_{i=1}^{n} k_{i} f_{i}(z) \text {. }
$$

Здесь $f_{i}(z)$ - линейно независимые, строго монотонные на $[a, b]$ функции (компоненты гидродинамического поля), а все коэффициенты $k_{l}(l=0,1, \ldots, n)$ отличны от нуля. И пусть $z_{1}-$ нулевая точка исследуемого поля $F$. Тогда по определению нулевой точки $F\left(z_{1}\right)=0$, или в силу представления (8)

$$
k_{0}+k_{1} f_{1}\left(z_{1}\right)+\ldots+k_{n} f_{n}\left(z_{1}\right)=0
$$

Получившееся уравнение (9) можно рассматривать как однородное уравнение с постоянными коэффициентами $f_{i}\left(z_{1}\right)$ относительно неизвестных значений $k_{i}$. Если у поля $F$ в слое $(a, b)$ несколько таких нулей (обозначим их $\left.z_{j}(j=1, \ldots, m)\right)$, то аналогичным образом получим систему $m$ однородных уравнений с постоянными коэффициентами $f_{i}\left(z_{j}\right)$ относительно параметров $k_{i}$ : 


$$
\begin{gathered}
k_{0}+k_{1} f_{1}\left(z_{1}\right)+\ldots+k_{n} f_{n}\left(z_{1}\right)=0, \\
\ldots \\
k_{0}+k_{1} f_{1}\left(z_{j}\right)+\ldots+k_{n} f_{n}\left(z_{j}\right)=0, \\
\ldots \\
k_{0}+k_{1} f_{1}\left(z_{m}\right)+\ldots+k_{n} f_{n}\left(z_{m}\right)=0 .
\end{gathered}
$$

Поскольку функции $f_{i}$ - строго монотонные функции, то согласно определению монотонности для любых $z_{1} \neq z_{2}$ выполняется неравенство $f_{i}\left(z_{1}\right) \neq f_{i}\left(z_{2}\right)$. Возьмем теперь два любых уравнения системы (10) и рассмотрим отношение коэффициентов, стоящих перед коэффициентами $k_{0}$ и $k_{1}$ :

$$
\frac{f_{1}\left(z_{j_{1}}\right)}{f_{1}\left(z_{j_{2}}\right)} \neq 1\left(j_{1}, j_{2}=1, \ldots, m ; j_{1} \neq j_{2}\right) \text {. }
$$

Из этого неравенства следует, что все уравнения системы (10) линейно независимы.

Если число $m$ нулевых точек (равное числу уравнений системы (10)) меньше числа $n+1$ неизвестных параметров $k_{i}$, то система (10) недоопределена. Ее можно рассматривать как $m$ связей, накладываемых на неизвестные коэффициенты $k_{0}, k_{1}, \ldots, k_{n}$. Следовательно, получаем $n-m+1$ независимых переменных. Задавая им произвольные значения, можно вычислить значения оставшихся $m$ переменных, чтобы удовлетворить системе (10).

Если $m>n+1$, то система переопределена, и она не имеет решения из-за линейной независимости уравнений рассматриваемой системы.

Если $m=n+1$, то система (10) становится системой $n+1$ однородного уравнения относительно $n+1$ коэффициента $\left(k_{0}, k_{1}, \ldots, k_{n}\right)$. Так как строки определителя системы линейно независимы, то он не обращается в нуль [43]. Следовательно, решение единственно, и это есть тривиальное решение. В этом случае точное решение для поля $F$ описывает тождественно нулевое поле, что противоречит базовому предположению.

Таким образом, у однородного поля $F$ внутри слоя $a \leq z \leq b$ может быть не более $n$ нулевых точек. А следовательно, не более $n$ точек стратификации.

Заметим, что непосредственно из этих рассуждений следует достижимость данной оценки. Действительно, если $m=n$, то система (10) есть система $n$ линейно-независимых уравнений относительно $n+1$ неизвестных коэффициентов $k_{0}, k_{1}, \ldots, k_{n}$. Такая система всегда имеет нетривиальное решение. Следовательно, всегда существует такой набор коэффициентов $k_{0}, k_{1}, \ldots, k_{n}$, что поле $F$ имеет $n$ нулевых точек внутри исследуемого слоя $[a, b]$. При этом значения поля $F$ на границах слоя (в точках $a$ и $b$ ) не оказывают никакого влияния на достижимость построенной оценки. 


\section{4. Анализ точного решения}

Проиллюстрируем метод, изложенный выше, на примере анализа скорости

$$
V=\frac{g \beta c_{1} c_{4}}{2520 v \chi} z^{7}+\frac{g \beta\left(c_{2} c_{4}+c_{1} c_{5}\right)}{720 v \chi} z^{6}+\frac{g \beta c_{2} c_{5}}{120 v \chi} z^{5}+\frac{g \beta c_{6}}{24 v} z^{4}+\frac{g \beta c_{7}}{6 v} z^{3}+\frac{c_{8}}{2 v} z^{2}+c_{9} z+c_{10}
$$

из точного решения (7). По теореме Гаусса [22] многочлен $V(z)$ как полином седьмой степени имеет не более семи действительных нулей, следовательно, у скорости $V$ не может быть более семи застойных точек. Этот же результат можно получить, опираясь на описанный выше подход. Обозначим

$$
\begin{gathered}
f_{i}(z)=z^{i}(i=1, \ldots, 7) ; \\
k_{0}=c_{10} ; k_{1}=c_{9} ; k_{2}=\frac{c_{8}}{2 v} ; k_{3}=\frac{g \beta c_{7}}{6 v} ; \\
k_{4}=\frac{g \beta c_{6}}{24 v} ; k_{5}=\frac{g \beta c_{2} c_{5}}{120 v \chi} ; k_{6}=\frac{g \beta\left(c_{2} c_{4}+c_{1} c_{5}\right)}{720 v \chi} ; k_{7}=\frac{g \beta c_{1} c_{4}}{2520 v \chi} .
\end{gathered}
$$

Несложно убедиться, что производная

$$
f^{\prime}(z)=\left\{\begin{array}{c}
i z^{i-1}, i=2, \ldots, 7 \\
1, i=1
\end{array}\right.
$$

может обращаться в нуль только в точке $z=0$ (для $i=2, \ldots, 7$ ) или не имеет нулей вообще (если $i=1$ ). Следовательно, функции $f_{i}(z)$ являются строго монотонными на интервале $(0, h)$ и оказываются справедливы выводы, сделанные при описании подхода к построению оценки на число нулевых точек произвольного однородного поля. Откуда незамедлительно следует, что в предельном случае, когда задаваемые граничные условия таковы, все коэффициенты $k_{0}, \ldots, k_{7}$ оказываются отличными от нуля, у компоненты $V_{y}=V(z)$ вектора скорости $\boldsymbol{V}$ может быть не более семи застойных точек. Предложенный подход применительно к общему решению (7) для скорости $V$ дает тот же результат, что и известные методы, но оказывается более полезным при рассмотрении именно краевых задач.

Замечание 1. Полученная выше оценка на число возможных критических точек исследуемого поля справедлива не только для задач конвекции вертикально завихренной жидкости, данный подход может быть применен и к исследованию течений, для которых вертикальная компонента завихренности равна нулю.

Пример 1. Полагая в (4) $u=0$, получим однородное поле скоростей

$$
V_{x}=U, V_{y}=V \text {. }
$$

В [7] для данного класса было получено точное решение, описывающее конвективные движения в бесконечном горизонтальном слое, вызванные неравномерным нагревом (охлаждением) границ слоя и заданием поля касательных напряжений на верхней его границе. В этом случае функция фоновой температуры $T_{0}$ после обезразмеривания принимала вид: 


$$
T_{0}(Z)=\frac{\delta P e}{2520} f(Z) Z
$$

где $f(Z)=15 Z^{6}-77 Z^{5}+147 Z^{4}+(a-105) Z^{3}-2 a Z^{2}+b(a, b-$ безразмерные параметры задачи); $P e=\frac{g \beta B h^{4}}{\chi v}-$ число Пекле; $\delta=h / l-$ показатель геометрической анизотропии; $Z=z / h-$ безразмерная вертикальная координата; $h, l$ - характерные вертикальный и горизонтальный размеры слоя соответственно. Полином шестой степени $f(Z)$ есть линейная комбинация функций

$$
f_{1}(Z)=15 Z^{6}-77 Z^{5}+147 Z^{4}-105 Z^{3} \text { и } f_{2}(Z)=Z^{3}-2 Z^{2}
$$

каждая из которых, является строго монотонной на интервале $(0,1)$. Таким образом, согласно описанному выше подходу, функция $f(Z)$ может иметь не более двух нулей. Следовательно, функция фоновой температуры $T_{0}$ допускает не более двух нулевых точек, что и было проиллюстрировано в [7] (рис. 1).

Замечание 2. При рассмотрении различных краевых условий может оказаться, что построенная для общего решения (7) оценка на число критических точек будет недостижима. Это может произойти по нескольким причинам: во-первых, часть из коэффициентов $k_{l}$ могут обратиться в нуль, соответственно уменьшится число слагаемых в представлении решения (8); во-вторых, при подстановке конкретных значений констант, определяющих конкретную жидкость, может получиться такая ситуация, что в разложении (8) найдутся два или более слагаемых, сумму которых можно представить в виде многочлена вида $k f(z)$, что также приведет к уменьшению числа членов ряда (8). И нет оснований считать, что этими ситуациями исчерпывается круг причин, по которым в конкретной краевой задаче число застойных точек какого-то поля будет меньше, чем в случае общего подхода, представленного выше. Для наглядной иллюстрации приведенных рассуждений рассмотрим следующую краевую задачу.

Пример 2. В качестве граничных условий для общего решения (7) рассмотрим систему условий, описывающую влияние термокапиллярного эффекта, задаваемого на верхней границе $z=h$ бесконечного горизонтального слоя, на свойства потока жидкости $[9,10]$. Будем полагать, что абсолютно твердая нижняя поверхность $z=0$ является отсчетным уровнем измерения температуры:

$$
T(x, y, 0)=0 .
$$

Будем полагать, что задана скорость движения нижней границы $[13,14]$

$$
V_{x}(x, y, 0)=W \cos \alpha+\Omega y ; V_{y}(x, y, 0)=W \sin \alpha .
$$

Верхнюю (свободную) поверхность $z=h$ считаем базовым уровнем отсчета приведенного давления. Полагаем, что задано однородное давление, совпадающее с атмосферным давлением, и по аналогии с заданием температуры принято за нуль:

$$
P(x, y, h)=0 \text {. }
$$


Будем также полагать, что на верхней границе задан термокапиллярный эффект:

$$
\eta \frac{\partial V_{x}}{\partial z}=-\sigma \frac{\partial T}{\partial x} ; \eta \frac{\partial V_{y}}{\partial z}=-\sigma \frac{\partial T}{\partial y}
$$

здесь $\eta$ - коэффициент динамической вязкости; $\sigma$ - температурный коэффициент поверхностного натяжения. Кроме того, на обеих границах слоя жидкости заданы источники теплового возмущения:

$$
T(x, y, 0)=B y ; T(x, y, h)=\vartheta+C x \text {. }
$$

С учетом структуры обобщенного решения (4) и (5) выбранные условия записываются в виде следующих равенств:

- на нижней границе слоя жидкости:

$$
u(0)=\Omega ; T_{0}(0)=T_{1}(0)=0 ; T_{2}(0)=B ; U(0)=W \cos \alpha ; V(0)=W \sin \alpha ;
$$

- на верхней границе слоя:

$$
\begin{gathered}
u^{\prime}(h)=0 ; T_{0}(h)=\vartheta ; T_{1}(h)=C ; T_{2}(h)=0 ; P_{0}(h)=P_{1}(h)=P_{2}(h)=0 ; \\
\eta U^{\prime}(h)=-\sigma T_{1}(h) ; \eta V^{\prime}(h)=-\sigma T_{2}(h) .
\end{gathered}
$$

Определяя с помощью системы условий (11) и (12) значение постоянных интегрирования $c_{i}(i=1, \ldots, 12)$ в точном решении (7), получим частное решение. После перехода к безразмерной координате $Z=z / h \in[0,1]$ компонента $V$ принимает вид:

$$
V=-\frac{b G r \delta^{3}}{12 T a} Z(Z-2)\left(Z^{2}-2 Z+2\right)+\frac{\delta^{5} P e}{720} Z\left(Z^{5}-5 Z^{3}+15 Z-16\right)+\frac{2 \operatorname{Recos} \alpha}{T a},
$$

где $G r=\frac{C g \beta l^{4}}{v^{2}} ; P e=\frac{W l}{\chi} ; R e=\frac{W l}{v} ; T a=\frac{2 \Omega l^{2}}{v}-$ числа Грасгофа, Пекле, Рейнольдса и модифицированное число Тейлора соответственно; $b=B / A-$ безразмерный параметр.

Скорость $V$ течения жидкости в направлении оси $O y$, как видно из (13), после подстановки краевых условий (11) и (12) в общее решение (7) остается полиномом шестой степени, следовательно, не может иметь более шести нулей. С другой стороны, оба многочлена, входящие в выражение (13) скорости $V$, являются строго монотонными внутри слоя [0,1]. Таким образом, все необходимые условия оказываются выполнены. В результате чего получаем оценку, согласно которой в данном конкретном случае скорость $V$, определяемая выражением (13), допускает наличие не более двух критических точек. На рис. 2 представлены примеры соответствующих течений. 


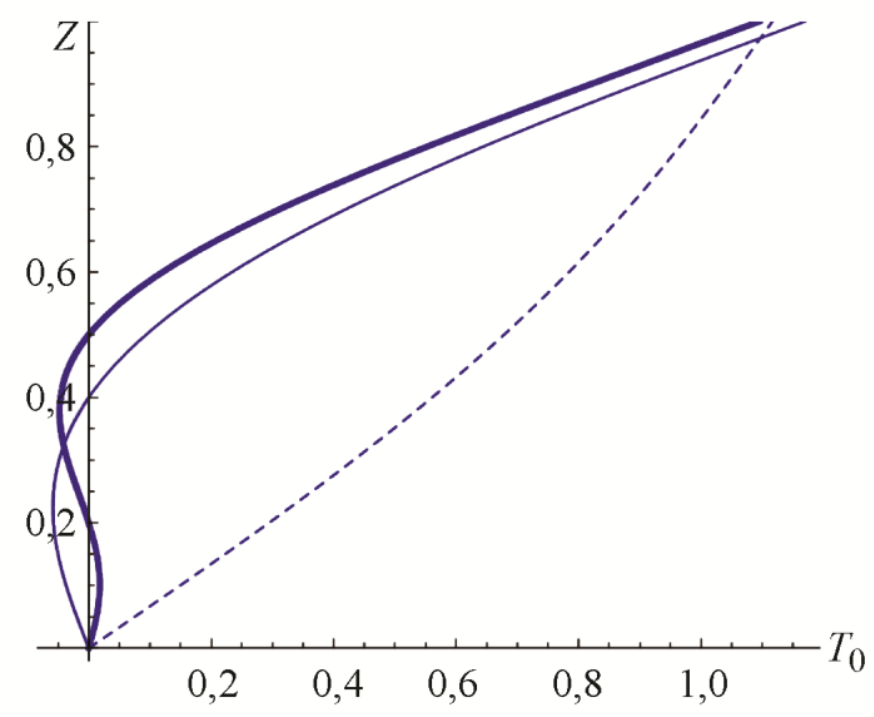

Рис. 1. Профили фоновой температуры $T_{0}$ при различном числе нулевых точек в рассматриваемом слое жидкости

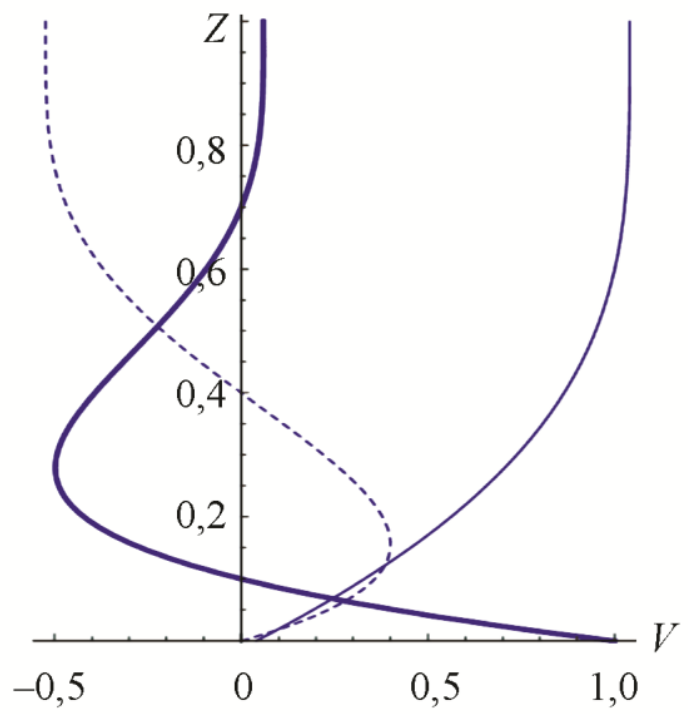

Рис. 2. Профили скорости $V$ при различном числе застойных точек

Замечание 3. В некоторых ситуациях при построении оценок на возможное число критических точек помимо изложенного выше подхода следует учитывать дополнительные ограничения, вызванные спецификой решения. Такие ограничения могут обосновываться, например, зависимостью одних параметров краевой задачи от других ее параметров.

Пример 3. Для краевой задачи, описанной в Примере 2, рассмотрим получившееся точное решение для поля давления. Его однородная составляющая определяется выражением:

$$
\begin{gathered}
P_{0}=\frac{g \beta \vartheta h\left(-239500800+239500800 Z^{2}\right)}{479001600}+ \\
+\frac{B^{2} g^{2} \beta^{2} h^{6}\left(653400-1900800 Z^{2}+3326400 Z^{4}-3326400 Z^{5}+1663200 Z^{6}-475200 Z^{7}+59400 Z^{8}\right)}{479001600 v \chi}+ \\
+\frac{C^{2} g^{2} \beta^{2} h^{6}\left(2174040-3896640 Z^{2}+2661120 Z^{5}-997920 Z^{6}+59400 Z^{8}\right)}{479001600 v \chi}+ \\
+\frac{C^{2} g \beta \sigma h^{4}\left(-11975040+19958400 Z^{2}-7983360 Z^{5}\right)}{479001600 \eta \chi}+ \\
+\frac{B C g^{2} \beta^{2} \Omega h^{8}}{479001600 v \chi^{2}}\left(-177804+449460 Z^{2}-443520 Z^{4}+121968 Z^{5}+83160 Z^{6}-15840 Z^{7}-\right. \\
\left.-39600 Z^{8}+27720 Z^{9}-5544 Z^{10}\right)+ \\
+\frac{B C g^{2} \beta^{2} \Omega h^{8}\left(-305844+531300 Z^{2}-266112 Z^{5}+63360 Z^{7}-29700 Z^{8}+7920 Z^{9}-924 Z^{10}\right)}{479001600 v^{2} \chi}+ \\
+\frac{C^{2} g^{2} \beta^{2} \Omega^{2} h^{10}\left(12036-25056 Z^{2}+29568 Z^{5}-13860 Z^{6}-8448 Z^{7}+6600 Z^{8}-924 Z^{10}+84 Z^{12}\right)}{479001600 v \chi^{3}}+
\end{gathered}
$$




$$
\begin{gathered}
+\frac{C^{2} g^{2} \beta^{2} \Omega^{2} h^{10}\left(49886-86536 Z^{2}+42768 Z^{5}-8448 Z^{7}+2475 Z^{8}-154 Z^{10}+9 Z^{12}\right)}{479001600 v^{2} \chi^{2}}+ \\
+\frac{C g \beta h^{3} W \cos \alpha\left(19958400-39916800 Z^{2}+19958400 Z^{4}\right)}{479001600 \chi}+ \\
+\frac{B g \beta h^{3} W \sin \alpha\left(19958400-79833600 Z^{2}+79833600 Z^{3}-19958400 Z^{4}\right)}{479001600 \chi}+ \\
+\frac{C g \beta \Omega h^{5} W \sin \alpha\left(-1995840+4656960 Z^{2}-3326400 Z^{4}+665280 Z^{6}\right)}{479001600 \chi^{2}}+ \\
+\frac{C g \beta \Omega h^{5} W \sin \alpha\left(-7983360+13970880 Z^{2}-7983360 Z^{5}+1995840 Z^{6}\right)}{479001600 v \chi} .
\end{gathered}
$$

Многочлен $P_{0}(Z)$ в выражении (14) является полиномом десятой степени, следовательно, число его нулей не превосходит десяти. С другой стороны, этот полином представлен в виде суммы постоянного слагаемого и линейной комбинации одиннадцати многочленов различной степени. При рассмотрении конкретной жидкости число независимых параметров (значения продольных градиентов температуры $B, C$; пространственное ускорение $\Omega$; толщина слоя $h$; параметры скорости движения нижней границы $W$ и $\alpha$ ) значительно меньше одиннадцати, значит, не все из входящих в выражение (14) полиномов будут независимы. Кроме того, два из этих одиннадцати многочленов не являются строго монотонными внутри слоя $(0,1)$, поэтому применить оценку, построенную на основе предложенного выше подхода, формально не удастся. В таких случаях необходимо проводить дополнительное исследование, например использовать связь фоновой температуры $T_{0}$ и фонового давления $P_{0}$, определяемую уравнением

$$
P_{0}^{\prime}=g \beta T_{0}
$$

системы (6). Согласно этому уравнению, число нулевых точек температуры $T_{0}$ совпадает с числом экстремумов функции $P_{0}$, а значит число нулевых точек фонового давления отличается от числа нулевых точек фоновой температуры не более чем на один.

\section{5. Заключение}

В статье на примере уравнений тепловой конвекции рассмотрен один подход к построению оценки на максимально возможное число нулевых точек исследуемых гидродинамических полей. Справедливость построенной оценки обосновывается доказательством соответствующего утверждения. Данный подход проиллюстрирован на примере трех краевых задач, описывающих конвективные движения в слоистых потоках вязкой несжимаемой жидкости при задании различных граничных условий. Ввиду того, что приведенное в статье доказательство вспомогательного утверждения не апеллирует никаким образом к механической интерпретации изучаемого явления, имеются основания утверждать, что данная методика применима не только к различным по физической трактовке процессам в механике жидкости, но и процессам, изучаемым в рамках некоторых разделов математической физики; причем эти процессы могут описываться нелинейными функциями общего вида. 


\section{Литература}

1. Наливкин Д. В. Ураганы, бури и смерчи. - Л. : Наука, 1969. - 487 с.

2. Океанология. Физика океана. Т. І. Гидрофизика океана / под ред. А. С. Монина и В. М. Каменковича. - М. : Наука, 1978. - 466 с.

3. Алексеенко С. В., Куйбин П. А., Окулов В. Л. Введение в теорию концентрированных вихрей. - Новосибирск : Институт теплофизики им. С. С. Кутателадзе, 2003. -504 с.

4. Лаврентьев М. А., Шабат Б. В. Проблемы гидродинамики и их математические модели. - 2-е изд. - М. : Наука, 1973. - 417 с.

5. Бурмашева Н. В., Просвиряков Е. Ю. Крупномасштабная слоистая стационарная конвекция вязкой несжимаемой жидкости под действием касательных напряжений на верхней границе. Исследование поля скоростей // Вестн. Сам. гос. техн. ун-та. Сер. Физ.-мат. науки. 2017. - T. 21, № 1. - C. 180-196. - DOI: 10.14498/vsgtu1527.

6. Шварц К. Г. Плоскопараллельное адвективное течение в горизонтальном слое несжимаемой жидкости с внутренним линейным источником тепла // Прикладная математика и механика. - 2018. - Т. 82, № 1. - С. 25-30.

7. Burmasheva N. B., Prosviryakov E. Yu. Exact solution for the layered convection of a viscous incompressible fluid at specified temperature gradients and tangential forces on the free boundary // AIP Conference Proceedings. - 2017. - Vol. 1915, iss. 1. - DOI: 10.1063/1.5017353.

8. Burmasheva N. V., Prosviryakov E. Yu. Convective layered flows of a vertically whirling viscous incompressible fluid. Velocity field investigation // Вестн. Сам. гос. техн. ун-та. Сер. Физ.-мат. науки. - 2019. - Т. 23, № 2. - С. 341-360. - DOI: 10.14498/vsgtu1670.

9. Burmasheva N. B., Prosviryakov E. Yu. Investigation of a velocity field for the Marangoni shear convection of a vertically swirling viscous incompressible fluid // AIP Conference Proceedings. - 2018. - Vol. 2053, iss. 1. - DOI: 10.1063/1.5084449.

10. Бекежанова В. Б., Родионова А. В. О длинноволновой устойчивости двухслойного течения жидкости по наклонной плоскости // Известия Российской академии наук. Механика жидкости и газа. - 2015. - № 6. - С. 3-19.

11. Андреев В. К., Бекежанова В. Б. О решении со свободным параметром уравнений конвекции в вертикальном цилиндре при объемном прогреве // Прикладная математика и механика. - 2013. - Т. 77, № 6. - С. 832-841.

12. Burmasheva N. B., Larina E. A., Prosviryakov E. Yu. Unidirectional convective flows of a viscous incompressible fluid with slippage in a closed layer // AIP Conference Proceedings. 2019. - Vol. 2176, iss. 1. - DOI: 10.1063/1. 5135147.

13. Бурмашева Н. В., Просвиряков Е. Ю. Исследование температурного поля в слоистых течениях вертикально завихренной вязкой несжимаемой жидкости при задании двух термокапиллярных сил на свободной границе // DReaM. - 2019. - № 1. - C. 6-42. DOI: 10.17804/2410-9908.2019.1.006-042.

14. Бурмашева Н. В., Просвиряков Е. Ю. Термокапиллярная конвекция вертикально завихренной жидкости // Теоретические основы химической технологии. $-2020 .-$ Т. 54, № $1 .-$ C. 114-124. - DOI: 10.31857/S0040357119060034.

15. Бекежанова В. Б. Конвективная неустойчивость течения Марангони-Пуазейля при наличии продольного градиента температуры // Прикладная механика и техническая физика. 2011. - Т. 52, № 1 (305). - С. 92-100.

16. Бурмашева Н. В., Просвиряков Е. Ю. Точное решение уравнений Навье-Стокса, описывающее пространственно неоднородные течения вращающейся жидкости // Труды Института математики и механики УрО РАН. - 2020. - Т. 26, вып. 2. - С. 79-87.

17. Бирих Р. В., Пухначев В. В., Фроловская О. А. Конвективное течение в горизонтальном канале с неньютоновской реологией поверхности при нестационарном продольном градиенте температуры // Известия Российской академии наук. Механика жидкости и газа. 2015. - № 1. - C. 192-198. 
18. Бурмашева Н. В., Просвиряков Е. Ю. Класс точных решений для двумерных уравнений геофизической гидродинамики с двумя параметрами Кориолиса // Известия Иркутского государственного университета. Серия Математика. - 2020. - Т. 32. - С. 33-48. DOI: $10.26516 / 1997-7670.2020 .32 .33$.

19. Гончарова О. Н. Двухслойные течения жидкостей с испарением на границе раздела при наличии аномального термокапиллярного эффекта // Известия Алтайского государственного университета. - 2015. - № 1-2 (85). - C. 101-105. - DOI 10.14258/izvasu(2015)1.218.

20. Александров А. Д., Колмогоров А. Н., Лаврентьев М. А. Математика, ее содержание, методы и значение. Т. 1. - М. : Издательство Академии наук СССР, 1956. - 536 с.

21. Шафаревич И. Р. О решении уравнений высших степеней (метод Штурма). - М. : Гостехиздат, 1954. - $24 \mathrm{c.}$

22. Башмакова И. Г. О доказательстве основной теоремы алгебры // Историко-математические исследования / под ред. Г. Ф. Рыбкина, А. П. Юшкевича. - М. : ГИТТЛ, 1957. - 820 с. Вып. 10.

23. Демидович Б. П. Лекции по математической теории устойчивости : учебное пособие. Санкт-Петербург : Лань, 2008. - 480 с.

24. Петров А. Г. Точное решение уравнений Навье-Стокса в слое жидкости между двумя движущимися параллельно пластинами // Прикладная механика и техническая физика. 2012. - Т. 53, № 5. - С. 13-18.

25. Шмыглевский Ю. Д. Аналитические исследования динамики жидкости и газа. М. : Эдиториал УРСС, 1999. - 232 с.

26. Кочин Н. Е., Кибель И. А., Розе Н. В. Теоретическая гидродинамика. Ч. 2. М. : ФИЗМАТЛИТ, 1963. - 728 с.

27. Лойцянский Л. Г. Механика жидкости и газа. - М. : ДРОФА, 2003. - 840 с.

28. Shvarts K. G. Advective Flow of a rotating fluid layer in a vibrational field // Russian Journal of Nonlinear Dynamics. - 2019. - Vol. 15, no. 3. - P. 261-270. - DOI: 10.20537/nd190305.

29. Аристов С. Н., Шварц К. Г. Адвективное течение во вращающейся жидкой пленке // Прикладная механика и техническая физика. - 2016. - Т. 57, № 1 (335). - С. 216-223. DOI: $10.15372 / \mathrm{PMTF} 20160121$.

30. Гершуни Г. 3., Жуховицкий Е. М. Конвективная устойчивость несжимаемой жидкости. - М. : Наука, 1972. - 392 с.

31. Ландау Л. Д., Лифшиц Е. М. Теоретическая физика. Т. 6. Гидродинамика. М. : ФИЗМАТЛИТ, 2001. - 736 с.

32. Boussinesq J. Theorie analitique de la chaleur. Vol. 2. - Paris : GauthierVillars, 1903. $625 \mathrm{p}$.

33. Oberbeck A. Uber die warmeleitung der flussigkeiten bei der berucksichtigung der stromungen infolge von temperaturdifferenzen // Ann. Phys. Chem. - 1879. - Vol. 7. - P. 271-292.

34. Aristov S. N., Prosviryakov E. Yu. Nonuniform convective Couette flow // Fluid Dynamics. 2016. - Vol. 51, no. 5. - P. 581-587. - DOI: 10.1134/S001546281605001X.

35. Аристов С. Н., Просвиряков Е. Ю. О слоистых течениях плоской свободной конвекции // Нелинейная динамика. - 2013. - Т. 9, № 4. - С. 651-657. - DOI: 10.20537/nd1304004.

36. Зайцев М. Л., Аккерман В. Б. Гипотеза об упрощении переопределенных систем дифференциальных уравнений и ее применение к уравнениям гидродинамики // Вестник ВГУ. Серия: Физика. Математика. - 2015. - № 2. - С. 5-27.

37. Тихонов А. Н., Самарский А. А. Уравнения математической физики. - М. : Наука, 1966. $-742 \mathrm{c}$.

38. Пиров Р. Об условиях совместности и многообразиях решений некоторых классов переопределенных систем дифференциальных уравнений в частных производных второго порядка // Уфимский математический журнал. - 2016. - Т. 8, № 2. - С. 59-65. 
39. Тарг С. М. Кориолиса сила // Физическая энциклопедия / гл. ред. А. М. Прохоров. М. : Советская энциклопедия, 1990. - 704 с. - Т. 2.

40. Coriolis G. Sur les equations du mouvement relative des systemes de corps // J. de 1'Ecole Polytechnique. - 1835. - Vol. 15, no. 24. - P. 142-154.

41. Manuel Lopez-Mariscal. Further Coriolis correlation considerations // Physics Today. 2012. - Vol. 65, iss. 11. - P. 8-9. - DOI: 10.1063/PT.3.1764.

42. Graney Ch. M. Coriolis effect, two centuries before Coriolis // Physics Today. - 2011. Vol. 64, iss. 8. - P. 8-9. - DOI: 10.1063/PT.3.1195.

43. Тыртышников Е. Е. Матричный анализ и линейная алгебра. - М. : МГУ, 2005. - 372 с. 